\title{
DECORIN INCREASES SURVIVAL AND AMELIORATES DISEASE PHENOTYPE OF COL7A1 HYPOMORPHIC MICE
}

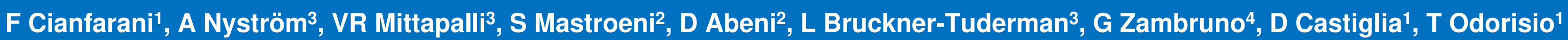

1. Lab. Molecular and Cell Biology, and 2. Clinical Epidemiology Unit, IDI-IRCCS, Rome, Italy; 3. Dept. Dermatology, University of Freiburg, Germany; 4. Genetic and Rare Diseases Research Area, Bambino Gesù Children's Hospital-IRCCS, Rome, Italy

\section{BACKGROUND}

- Recessive dystrophic epidermolysis bullosa (RDEB) is a rare skin blistering disease due to mutations in the gene COL7A1 coding for type VII collagen.

- In RDEB, unremitting skin and mucosal blistering leads to chronic wounds, inflammation and fibrosis; these features are responsible for more severe disease complications, such as pseudosyndactyly, esophageal stenosis and squamous cell cancer

- Recent studies highlighted the role of the TGF- $\beta$ pathway in modifying RDEB severity, and our previous findings showed that the proteoglycan decorin (DCN), an extracellular matrix component and a biological TGF- $\beta$ inhibitor, may influence disease outcome by counteracting TGF- $\beta$ profibrotic and proinflammatory activity (Odorisio et al, Hum Mol Gen 2014).

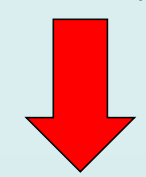

DCN appears as a potential therapeutic candidate to prevent or mitigate disease complications in patients affected with RDEB.

Figure 2

Increased survival of col7a1 hypomorphic mice treated with LV-DCN

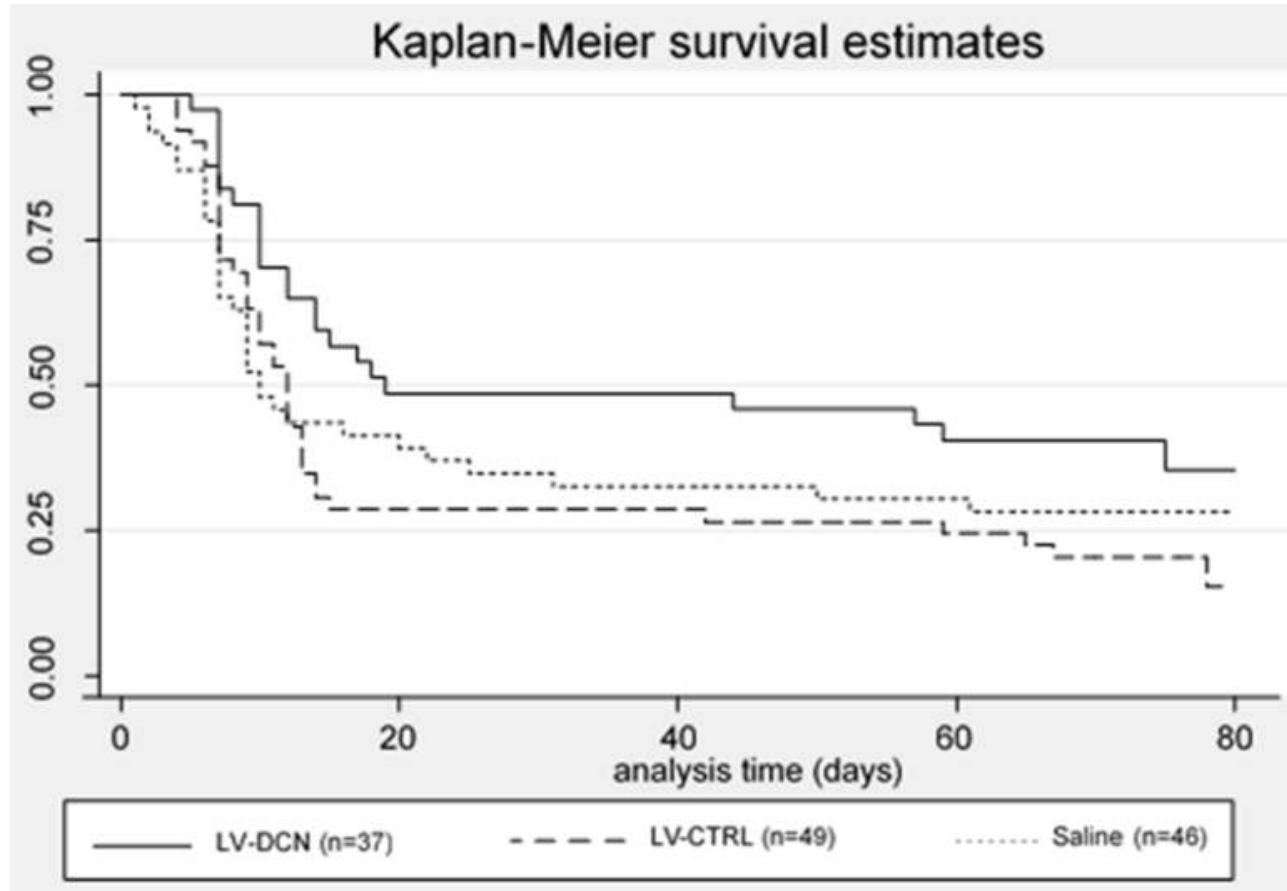

\begin{tabular}{|cc||ll|}
\cline { 2 - 4 } \multicolumn{2}{|c|}{ median survival time (12 days) } & \multicolumn{3}{|c|}{ survival rate $\mathbf{( 2 5 . 9 \% )}$} \\
LV-DCN & 19 days \\
LV-CTRL & 12 days \\
saline & 10 days \\
LV-DCN & $40.5 \%$ \\
LV-CTRL & $20.4 \%$ \\
saline & $28.3 \%$ \\
\hline
\end{tabular}

Pairwise comparison (Wilcoxon's test) $\mathrm{p}$-values for survival rate:

LV-DCN vs LV-CTRL, 0.02; LV-DCN vs Saline, 0.04; LV-CTRL vs Saline, 0.78

\section{Figure 4}

Reduced fibrosis in the skin of LV-DCN-treated mice
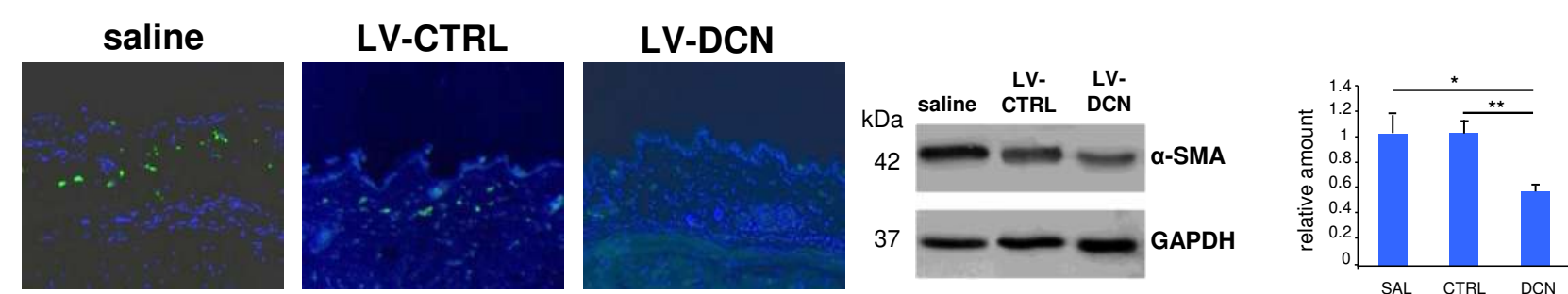

a-SMA (back skin)
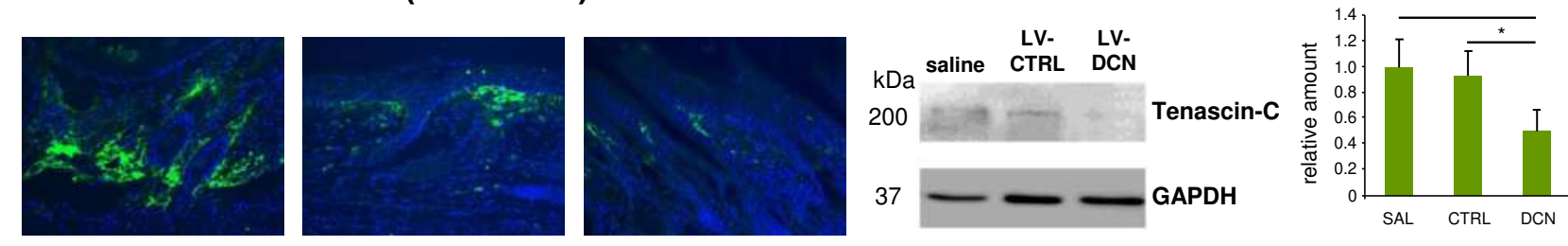

Tenascin-C (paws)
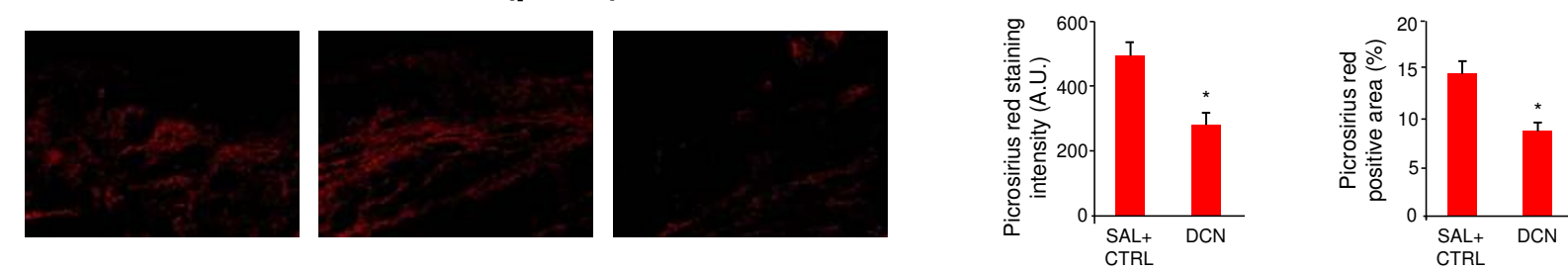

Picrosirius red staining (paws)

- As markers for tissue fibrosis, a-smooth muscle actin ( $\alpha$-SMA, expressed by myofibroblasts) and Tenascin-C (an extracellular matrix glycoprotein promoting fibrosis via Toll-like receptor 4 binding) were analyzed by immunofluorescence in forepaws, and by immunofluorescence and western blot in back skin $(n=$ saline 8; LV-CTRL 6; LV-DCN 9).

- The expression of $\alpha-S M A$ and Tenascin- $C$ was significantly reduced in both back skin and paws in LV-DCN treated mice as compared to controls.

- Staining by picrosirius red to visualize collagen networks/bundles under polarized light was reduced in skin samples from the forepaws of LV-DCN treated mice; the reduction was significant when control values were pooled and analyzed against LV-DCN values. However, this reduction was not evident in skin samples from back skin. $\left({ }^{*} p<0.05 ;{ }^{* *} p<0.01\right)$.

\section{AIM}

TO EVALUATE DECORIN POTENTIAL IN IMPROVING RDEB DISEASE PHENOTYPE BY USING A SEVERE RDEB

PRECLINICAL MODEL, THE col7a1 HYPOMORPHIC MOUSE

\section{Figure 1}

Lentiviral administration

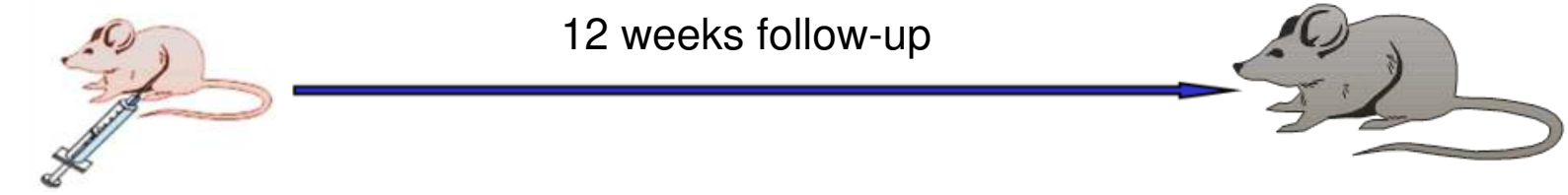

A single intraperitoneal treatment

between 7 and 12 days of age:

1) LV-DCN $\left(10^{7} T U\right)$

2) LV-CTRL (107TU)

3) saline solution

Survived mice were sacrified; back skin and forepaws were collected and analyzed

rention solution

ELISA for human decorin in the skin

Newborn Col7a1 hypomorhic mice show a great variability in disease manifestations. They were classified into four groups according to disease severity, and animals from each group were distributed homogeneously among the three treatments.

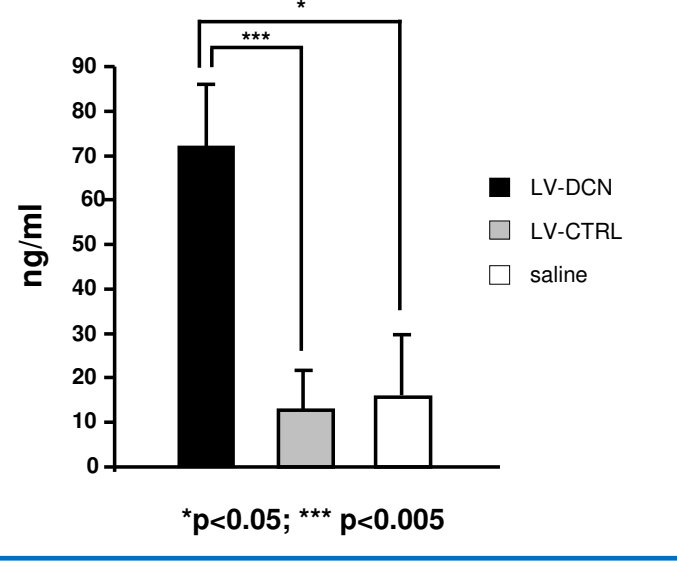

Figure 3

Increased digit length and reduced digit loss in LV-DCN-treated mice

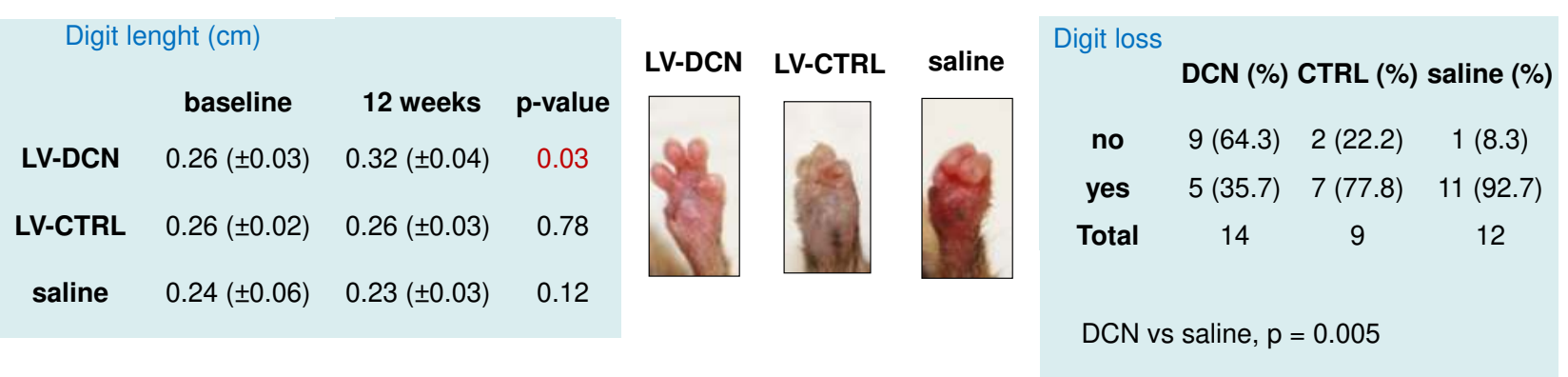

- At the endpoint, only col7a1 hypomorphic mice treated with LV-DCN exhibit a significant increase in digit length.

- A reduction in digit loss was observed in col7a1 hypomorphic mice treated with LV-DCN as compared to control treatments.

\section{Figure 5}

Reduced TGF- $\beta$ signaling in the skin of LV-DCN-treated mice

- TGF- $\beta$ signaling was analyzed by detecting the activation of SMAD2 (phosphorylated SMAD2, p-SMAD2).

- The analysis of back skin protein extracts by western blot revealed reduced amount of $\mathrm{p}$-SMAD2 in LV-DCN-treated mice as compared to controls $\left({ }^{*} \mathrm{p}<0.05\right)$

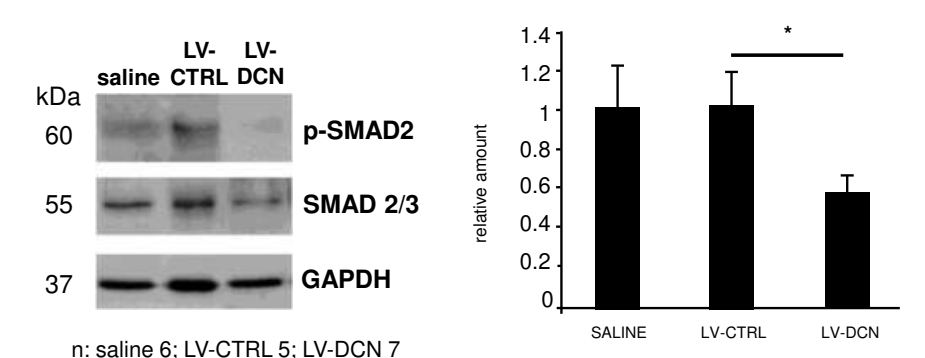

\section{CONCLUSIONS}

- By contrasting TGF- $\beta$-driven fibrosis, DCN is able to alleviate disease symptoms and increase survival rate of Col7a1 hypomorphic mice.

- Our data corroborate the results obtained by systemically treating col7a1 mice with losartan, a drug interfering with TGF- $\beta$ signaling (Nyström et al, EMBO Mol Med 2015). These findings advocate clinical testing of anti TGF- $\beta$ pathway agents for symptom-relief therapies in RDEB. 